\title{
Sistemas de Seguridade Social na América Latina: uma avaliação ordinal
}

\section{John Dixon}

Departamento de Política Social e Trabalho Social

University of Plymouth - Reino Unido

\begin{abstract}
Resumo
Este artigo amplia a literatura comparativa de sistemas de seguridade na América Latina através da incorporação da dimensão avaliativa. Seu objetivo é a classificação dos sistemas de seguridade nos 36 países latino-americanos utilizando uma metodologia que possibilita uma avaliação do modelo estatutário do sistema de cada país. A conclusão mostra que a região com os melhores modelos é a América Central, marginalmente à frente da América do Sul. Os melhores sistemas nacionais de seguridade na América Latina estão na Bolívia, Uruguai, Brasil, Nicarágua e Argentina. Observam-se programas de nível mundial no Brasil, Argentina, Chile, Peru e Nicarágua.

Palavras-chave: América Latina, seguridade social, metodologia de avaliação.

\section{Abstract}

This paper extends the comparative social security literature on Latin America by incorporating an evaluative dimension. The purpose of this paper is to rank social security systems in 36 Latin American countries using a comparative evaluation methodology that enables an assessment to be made of a country's statutory social security intention. The conclusion drawn is that the region with the best designed Latin American social security systems is Central America, very marginally ahead of South America. The best-designed national social security systems in Latin American be found in Bolivia, Uruguay, Brazil, Nicaragua and Argentina. World-class designed social security programs exist in Brazil, Argentina, Chile, Peru and Nicaragua.
\end{abstract}

Key words: Latin America, social security, evaluation methodology. 


\section{Introdução}

Na América Latina, as definições estatutárias da seguridade social, que datam das primeiras décadas do século $X X$, são dominadas pela estratégia de seguridade vinculada ao trabalho (ver Tabela 1).

Entretanto, há outras estratégias em funcionamento: contribuição do empregador, formas privatizadas de planos ocupacionais obrigatórios, planos pessoais obrigatórios, assistência social - contribuições sociais e fundos públicos obrigatórios -Fundos Nacionais Previdenciários (Anexo 1).

\section{TABELA 1}

\section{Estratégias de Seguridade Social}

\begin{tabular}{|c|c|c|c|c|c|}
\hline $\begin{array}{l}\text { Estratégia de } \\
\text { seguridade } \\
\text { social }\end{array}$ & Objetivo & $\begin{array}{l}\text { Fontes de } \\
\text { Financiamento }\end{array}$ & Cobertura & $\begin{array}{l}\text { Eligibilidade aos } \\
\text { Benefícios }\end{array}$ & $\begin{array}{l}\text { Formas de } \\
\text { Benefícios }\end{array}$ \\
\hline $\begin{array}{l}\text { Assistência } \\
\text { Social }\end{array}$ & $\begin{array}{l}\text { Redução da } \\
\text { pobreza }\end{array}$ & Orçamento público & $\begin{array}{l}\text { População total ou } \\
\text { categorias } \\
\text { designadas }\end{array}$ & $\begin{array}{l}\text { Domicílio } \\
\text { Testes } \\
\text { categóricos } \\
\text { Testes de médias }\end{array}$ & $\begin{array}{l}\text { Pagamentos } \\
\text { periódicos } \\
\text { Benefícios em } \\
\text { serviços }\end{array}$ \\
\hline Proteção Social & $\begin{array}{l}\text { Prevenção da } \\
\text { pobreza }\end{array}$ & $\begin{array}{l}\text { Contribuições dos } \\
\text { trabalhadores, seus } \\
\text { empregadores, e o } \\
\text { governo }\end{array}$ & $\begin{array}{l}\text { Trabalhadores em } \\
\text { categorias } \\
\text { designadas e seus } \\
\text { dependentes }\end{array}$ & $\begin{array}{l}\text { Testes } \\
\text { Categóricos } \\
\text { documento de } \\
\text { contribuição ou } \\
\text { emprego }\end{array}$ & 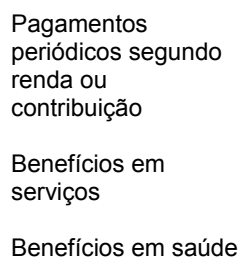 \\
\hline $\begin{array}{l}\text { Contribuições } \\
\text { Sociais }\end{array}$ & $\begin{array}{l}\text { Compensação } \\
\text { social }\end{array}$ & Orçamento público & $\begin{array}{l}\text { População total ou } \\
\text { categorias } \\
\text { designadas }\end{array}$ & $\begin{array}{l}\text { Testes } \\
\text { categóricos } \\
\text { Domicílio }\end{array}$ & $\begin{array}{l}\text { Pagamentos } \\
\text { períodicos }\end{array}$ \\
\hline $\begin{array}{l}\text { Recursos } \\
\text { Publicos } \\
\text { Obrigatórios }\end{array}$ & $\begin{array}{l}\text { Prevenção da } \\
\text { pobreza }\end{array}$ & $\begin{array}{l}\text { Contribuições de } \\
\text { empregados } \\
\text { cobertos e seus } \\
\text { empregadores }\end{array}$ & $\begin{array}{l}\text { Empregados de } \\
\text { categorias } \\
\text { designadas e seus } \\
\text { dependentes }\end{array}$ & $\begin{array}{l}\text { Testes } \\
\text { categóricos } \\
\text { Pagamento } \\
\text { passado de } \\
\text { contribuições }\end{array}$ & $\begin{array}{l}\text { Pagamentos globais, } \\
\text { talvez conversíveis } \\
\text { em pagamentos } \\
\text { periódicos }\end{array}$ \\
\hline $\begin{array}{l}\text { Impostos } \\
\text { Empregador } \\
\text { Pensões ou }\end{array}$ & $\begin{array}{l}\text { Prevenção da } \\
\text { pobreza }\end{array}$ & $\begin{array}{l}\text { Empregadores } \\
\text { designados }\end{array}$ & $\begin{array}{l}\text { Empregados e seus } \\
\text { dependentes }\end{array}$ & Emprego Atual & $\begin{array}{l}\text { Pagamentos } \\
\text { periódicos segundo } \\
\text { renda pagamentos } \\
\text { globais }\end{array}$ \\
\hline $\begin{array}{l}\text { Proventos } \\
\text { Legais } \\
\text { Ocupacionais }\end{array}$ & $\begin{array}{l}\text { Prevenção da } \\
\text { pobreza }\end{array}$ & $\begin{array}{l}\text { Contribuições de } \\
\text { empregados } \\
\text { cobertos e seus } \\
\text { empregadores }\end{array}$ & $\begin{array}{l}\text { Empregados de } \\
\text { categorias } \\
\text { designadas e seus } \\
\text { dependentes }\end{array}$ & $\begin{array}{l}\text { Pagamento das } \\
\text { últimas } \\
\text { contribuições }\end{array}$ & $\begin{array}{l}\text { Pagamentos globais } \\
\text { reajustados } \\
\text { pagamentos } \\
\text { periódicos ou } \\
\text { benefícios definidos }\end{array}$ \\
\hline $\begin{array}{l}\text { Pensões ou } \\
\text { Proventos } \\
\text { Pessoais } \\
\text { Legais }\end{array}$ & $\begin{array}{l}\text { Prevenção da } \\
\text { pobreza }\end{array}$ & $\begin{array}{l}\text { Contribuições de } \\
\text { segurados }\end{array}$ & $\begin{array}{l}\text { Contribuições } \\
\text { individuais }\end{array}$ & $\begin{array}{l}\text { Pagamentos das } \\
\text { últimas } \\
\text { contribuições }\end{array}$ & $\begin{array}{l}\text { Pagamentos globais } \\
\text { reajustados, } \\
\text { pagamentos } \\
\text { períodicos }\end{array}$ \\
\hline
\end{tabular}


Os primeiros estudos comparativos sobre provisão estatutária de seguridade social latino-americana emergiram no início dos anos 70 e foram marcados pelo trabalho seminal de Mesa-Lago ${ }^{1}$. Este estudo estende essa literatura através da incorporação de uma dimensão avaliativa que permite classificar os sistemas de seguridade social.

\section{Enfoques para Avaliação de Sistemas de Seguridade Social}

Uma variedade de metodologias de avaliação pode ser utilizada para avaliar sistemas de seguridade social (Dixon 1998). Inicialmente, poderiam ser apreendidos por seus 'inputs', como programas de gastos com idosos como porcentagem do PNB (ver, por exemplo, OECD 1976, 1977). Esse enfoque excluiria, sem dúvida, os programas financiados apenas por empregadores ou indivíduos, e uma cuidadosa apreensão da comparabilidade dos dados de gastos é obrigatória (OIT 1972b). Dessa forma, a metodologia de avaliação do insumo é problemática quando aplicada em comparação internacional em larga escala. Em segundo lugar, os programas poderiam ser julgados por seu desempenho (ou seja, eficiência ou efetividade) (ver, por exemplo, ISSA 1976). Uma avaliação de eficiência, usando como medida o custo administrativo por benefício disperso é problemática devido à dupla contagem e devido à existência de ausências causadas pelos serviços oferecidos por agências de múltiplos serviços (ex: correios). Uma avaliação de desempenho pode ter uma variedade de formas. Medidas de cobertura de programa podem ser utilizadas (como a porcentagem da população ou da força de trabalho cobertas), mas são problemáticas devido às dificuldades de definição do número de pessoas que realmente - mais que potencialmente- são elegíveis para os benefícios sob um critério geral de qualificação. Podem ser utilizadas medidas de adequação aos benefícios- como parâmetros de renda, como uma porcentagem da renda limítrofe da pobeza, salário mínimo ou salário médio, ou o PIB per capita, mas são ambíguas porque ignoram as necessidades diferenciais de seguridade e a distribuição de pagamento do benefício acima ou abaixo do padrão escolhido (como o limite de renda da pobreza, nível salarial médio, PIB per capita). Podem ser utilizadas medidas de percepção da satisfação das necessidades ou de adequação dos benefícios pelos beneficiários (tais como as medidas de acesso ao financiamento de habitação, ou medidas subjetivas de pobreza, todas, entretanto, possivelmente enviezadas pelo formato da questão aplicada e das escalas de medida utilizadas.

Além disso, dois desafios realmente formidáveis para avaliação de eficiência e desempenho permanecem. O primeiro é metodológico: como conciliar vários indicadores

\footnotetext{
${ }^{1}$ Mesa-Lago:ILO 1972a e 1993; Mesa-Lago:1978, 1983, 1985 a, 1985b, 1986, 1988, 1991 a, 1992a , 1992b, 1994,ver também Paillas:1979, Tamburi:1985, Dixon and Scheurell:1990, McGreevey:1990, World Bank:1995.
} 
complexos de avaliação em um único indicador que permita a elaboração de um ranking classificatório de sistemas nacionais de seguridade social?

O segundo é informacional: como superar a indisponibilidade de dados confiáveis e compatíveis especialmente em grandes bases internacionais? Enquanto uma metodologia avaliativa-comparativa de aplicação regional, as metodologias de eficiência e desempenho também são necessárias. Finalmente, programas de seguridade poderiam ser julgados segundo a adequação de seu modelo (Kaim-Caudle 1973). Este é o enfoque adotado neste estudo.

Uma metodologia comparativa de avaliação foi desenvolvida para dimensionar as características dos modelos de seguridade social. Isso possibilita uma avaliação da definição estatutária de um país com relação aos programas para idosos. O uso dessa metodologia também possibilita uma classificação dos sistemas nacionais de seguridade em 36 países da América Latina. Essa metodologia vai ao encontro do antigo desafio avaliativo-comparativo tratado por Rys (1966:268) de definir"escalas de classificação pelas quais julgar os méritos e resultados respectivos de membros individuais do universo [de seguridade social] observado".

Existe certamente a possibilidade de um hiato de implementação existente entre o que um sistema de seguridade promete oferecer - em termos do programa de cobertura, elegibilidade aos benefícios e generosidade dos benefícios e administração e finanças do programa - e o que ele oferece em última instância. Esse hiato pode, certamente, tornar-se muito significativo em países onde a administração pública ou as finanças públicas tenham larga ou totalmente falido, ou sido severamente restritas, em função de guerras, desastres naturais ou sérios desequilíbrios econômicos.

\section{Uma Metodologia de Avaliação}

Operacionalizar essa metodologia de avaliação de itens envolveu duas etapas. A primeira foi a articulação de um amplo conjunto de 860 itens característicos de sistemas de seguridade social (Tabela 2). A segunda foi a agregação sistemática de um 'score' subjetivo para a inclusão ou exclusão de cada item, de acordo com o fato de sua inclusão ou exclusão fazer tal sistema 'mais' ou 'menos' aceitável. Um conjunto de premissas valorativas é central a qualquer julgamento de avaliação qualitativa. As adotadas neste estudo relacionam o conjunto de parâmetros incorporados nas convenções da OIT sobre padrões mínimos de seguridade social (OIT 1952 a, 1952b, 1964, 1966, 1967, 1968, 1969, 1988). Essas convenções são duradouras e definem um conjunto internacionalmente aceito de padrões que identificam os itens que deveriam ser incorporados em um programa "minimamente aceitável" de seguridade para os idosos tanto em países desenvolvidos quanto em desenvolvimento (OIT 1989; Tamburi 1981). Oting (1993:169) considera que essas convenções oferecem "uma definição internacionalmente aceita da idéia de seguridade social". 
TABELA 2

Dimensões Avaliativas de Aspectos do Sistema de Seguridade Social

\begin{tabular}{|c|c|c|c|c|c|}
\hline $\begin{array}{l}\text { Estratégias Principais do } \\
\text { Sistema: }\end{array}$ & Cobertura & Elegibilidade & Benefícios & $\begin{array}{l}\text { Estratégias } \\
\text { Suplementares }\end{array}$ & $\begin{array}{c}\text { Componentes } \\
\text { Total }\end{array}$ \\
\hline $\begin{array}{l}\text { Programa de Auxílio a } \\
\text { Idosos }\end{array}$ & 21 & 18 & 32 & 3 & 74 \\
\hline $\begin{array}{l}\text { Programa de Auxílio- } \\
\text { Invalidez }\end{array}$ & 22 & 17 & 36 & 3 & 78 \\
\hline Aposentadorias & 23 & 39 & 33 & 3 & 98 \\
\hline $\begin{array}{l}\text { Programa de Auxílio- } \\
\text { doença }\end{array}$ & 27 & 10 & 19 & 3 & 59 \\
\hline $\begin{array}{l}\text { Programa de Auxílio- } \\
\text { maternidade }\end{array}$ & 25 & 13 & 35 & 3 & 76 \\
\hline $\begin{array}{l}\text { Programa de Auxílio } \\
\text { Temporário por Acidente } \\
\text { de Trabalho }\end{array}$ & 20 & 8 & 15 & 3 & 46 \\
\hline $\begin{array}{l}\text { Programa de Auxílio } \\
\text { Permanente por Acidente } \\
\text { de Trabalho }\end{array}$ & 21 & 10 & 32 & 3 & 66 \\
\hline $\begin{array}{l}\text { Aposentadoria relacionada } \\
\text { ao Trabalho }\end{array}$ & 20 & 29 & 40 & 3 & 92 \\
\hline $\begin{array}{l}\text { Programa de Auxílio } \\
\text { Desemprego }\end{array}$ & 12 & 15 & 34 & 3 & 64 \\
\hline $\begin{array}{l}\text { Programa de Auxílio- } \\
\text { Família }\end{array}$ & 24 & 10 & 19 & 3 & 56 \\
\hline $\begin{array}{l}\text { Programa de Benefícios a } \\
\text { Crianças }\end{array}$ & 24 & 18 & 39 & 3 & 84 \\
\hline $\begin{array}{l}\text { Programa de Benefícios } \\
\text { de Saúde }\end{array}$ & 13 & 2 & 14 & 4 & 84 \\
\hline Formas de Financiamento & & & & & 27 \\
\hline Formas de Administração & & & & & 7 \\
\hline TOTAL & 252 & 189 & 348 & 37 & 860 \\
\hline
\end{tabular}

Fonte: Dixon 1999: 200. 
As convenções da OIT abrangem as seguintes dimensões sistêmicas: requisitos de cobertura, critérios de eligibilidade ao benefício, provisões de benefícios, finanças e administração dos beneficios.

Os sistemas de seguridade social são considerados mais aceitáveis (em vários graus) se seus itens:

- Cobrem todas as contingências de seguridade social, o que penaliza países que escolheram políticas por razões ideológicas, políticas ou econômicas, ou todas:

- utilizando outras estratégias de políticas públicas (como estratégias de taxas de gasto) para obter objetivos de seguridade;ou

- não estabelecendo programas de seguridade para contingências particulares;

- Incorporam em seus programas constituintes:

- universalidade da cobertura, o que penaliza países que optaram pela cobertura restrita através da exclusão de segmentos específicos da população, por razões ideológicas, políticas ou econômicas;

- restrições mínimas com relação aos requisitos de elegibilidade de qualificação geral e segmentação, e a especificação de critério de necessidade, o qual penaliza os países que definem políticas de eligibilidade restritiva em bases diferentes, seja por

- provisão de pensões periódicas que capacita os beneficiários manterem seus estilos de vida, relativos aos padrões de vida prevalecentes da comunidade, o que penaliza países que definiram políticas, tanto por razões ideológicas, políticas ou econômicas, de:

- não provisão de benefícios segundo salários anteriores; ou

ajuste não regular de pensões, de forma a assegurar que permanceçam adequadas aos padrões de vida da comunidade

- provisão de benefícios de saúde que incluem atenção médica, hospitalar e paramédica, com um padrão comparável à atenção disponível na comunidade, àqueles cobertos pelos programas de seguridade social (incluindo dependentes) $\mathrm{e}$ àqueles beneficiários que necessitam desses serviços pelo tempo em que tais serviços são clinicamente necessários, o que penaliza os países que definem políticas de restrição de disponibilidade, ou da faixa de benefícios de saúde providas pelo sistema de seguridade; e

- provisão de incentivos para encorajar ou capacitar os recipientes do sistema de seguridade social que são capazes de ingressar na força de trabalho, o que penaliza países que definem políticas de não introdução de um conjunto de medida e de bem-estar no trabalho, no esforço de reduzir a dependência.

- Minimiza seus custos e divide-os entre empregadores, empregados e o governo de tal forma que assegura que os custos dirigidos aos indivíduos (contribuintes) seja progressivo e não regressivo, o que penaliza países que fizeram uma escolha de política, mesmo que por razões econômicas, políticas ou ideológicas, de : 
.não adotar financiamento tripartite para todos os programas de seguridade, ou .limitar o grau de redistribuição de renda vertical

- Tem um modo de administração que é o mais simples e decentralizado possível, especialmente pela perspectiva do usuário final, o que penaliza países que fizeram uma escolha de política, mesmo que por razões econômicas, políticas ou ideológicas, de construir um sistema de seguridade complexo ou centralizado.

Para cada sistema de seguridade social de cada país, o score de classificação(R) foi calculado da seguinte maneira:

$$
R=a[(\text { Psoma }+H) / 11]+b(F)+c(A)
$$

Onde,

- Psoma é a soma dos 'scores' de avaliação de desenho (P) dos dez programas nacionais de seguridade social (ver Tabela 2), onde para cada programa:

$$
P=0.3[(100-C d+C b)+(100-E d+E b)+(100-B d+B b)+S b],
$$

Onde, $C d$ é a soma de todas as deduções do programa de cobertura (como por exemplo, excluindo a cobertura obrigatória as pessoas sem emprego formal): $C b$ é a soma de todos os bônus de mérito do programa de cobertura (como por exemplo, tornar disponível a cobertura voluntária para os excluídos da cobertura obrigatória); Ed é a soma de todas as deduções do programa de eligibilidade de benefícios (como, por exemplo, excluindo da elegibilidade de benefícios aos incapazes qualquer um que é incapaz mas tem capacidade de perda de proventos menor que 100\%); Eb é a soma de todos os bônus de mérito do programa de eligibilidade (como por exemplo, incluindo provisões de aposentadoria tardia ou antecipada); $B d$ é a soma de deduções de benefícios (ex: para a provisão de apenas qualificações para pagamentos globais ou serviços isolados); $B b$ é a soma dos bônus de mérito (como a provisão de benefícios suplementares ou de necessidades especiais); e $S b$ é o bônus de mérito conferido por qualquer programa suplementar (por exemplo, estendendo a cobertura da população ou suplementando benefícios).

• H é o score nacional de avaliação dos serviços de saúde, onde:

$$
\mathrm{H}=0.3[(100-\mathrm{HCd}+\mathrm{HCb})+(100-\mathrm{Hed}+\mathrm{Heb})+(100-\mathrm{HBd}+\mathrm{HBb})+\mathrm{HS}]
$$

Onde, $H C d$ é a soma das deduções da cobertura de saúde (ex: excluindo da cobertura obrigatoria as pessoas sem emprego formal); $H C b$ é a soma dos bônus de mérito da cobertura de saúde ( ex: disponibilizando a cobertura voluntária para os excluídos da cobertura obrigatória); Hed é a soma das deduções de elegibilidade aos benefícios de saúde (ex: excluindo os dependentes da elegibilidade aos benefícios); Heb é a soma dos 
bônus de mérito de elegibilidade aos benefícios (ex: por inclusão de dependentes além dos dependentes diretos elegíveis aos benefícios de saúde); $H B d$ é a soma das deduções principais dos benefícios ( ex: limitação do tempo de tratamento em hospital); $H B b$ é a soma de todos os bônus de mérito do programa de benefícios em saúde (ex: inclusão de provisão aos serviços oftalmológicos); e HS é o bônus de mérito designado para quaisquer programas suplementares de saúde (ex: extensão da população com cobertura ou suplementação de benefícios em saúde);

• F é score nacional de avaliação de financiamento da seguridade social, onde:

$$
\mathrm{F}=100-\mathrm{Fd}+\mathrm{Fb}
$$

Onde, $F d$ é a soma de todas as deduções do plano de financiamento de seguridade social (ex: imposição de contribuições apenas por cada serviço ao empregador); e Fb é a soma de todos os bônus de mérito do plano de financiamento da seguridade social (ex: provisão de subsídios a um governo almejando grupos necessitados da população);

${ }^{\circ}$ A é score nacional de avaliação administrativa da seguridade social, onde:

$$
A=(100-A d+A b)
$$

Onde, $A d$ é a soma de todas as deduções do plano administrativo (ex: ter mais que 5 agências de oferta de programas de seguridade social), e $A b$ é a soma de todos os bônus de mérito do plano administrativo (ex: ter um sistema administrativo descentralizado); e

' a, b e c são coeficientes de importância relativa que compõem a unidade, tendo valores de $0.8,0.15$ e 0.05 , respectivamente.

O resultado é uma classificação regional da América Latina para sistemas nacionais de seguridade social (ver Anexo 2). 


\section{Uma Classificação dos Programas e Sistemas de Seguridade Social Latino-Americanos}

Os padrões globais obtidos pelos sistemas de seguridade na América Latina são mais altos que aqueles obtidos na África, Oriente Médio e Ásia, embora estejam consideravelmente abaixo dos obtidos na América do Norte, Europa e Australásia. O melhor sistema latino-americano-Bolívia- entretanto, é melhor que o melhor da África Tunísia- mas de um padrão mais baixo que melhores planos do Oriente Médio - Israel- ou Ásia - Armênia e Japão (Dixon:1999).

A classificação latino-americana é dominada pelos mais velhos sistemas de seguridade da América do Sul - Bolívia(1\%lugar), Uruguai ( $2^{\circ}$ lugar), Brasil (3lugar), Argentina ( $5^{\circ}$ lugar) e Chile ( $7^{\circ}$ lugar) - e América Central - Nicarágua (4\%lugar), México (6\%lugar) e Costa Rica ( $8 \%$ lugar), com as Bahamas (9\%ugar) e Trinidad e Tobago (10\% lugar) somo os únicos países do Caribe entre os dez primeiros classificados.

TABELA 3

Classificações de Seguridade Social para América Latina, 1995

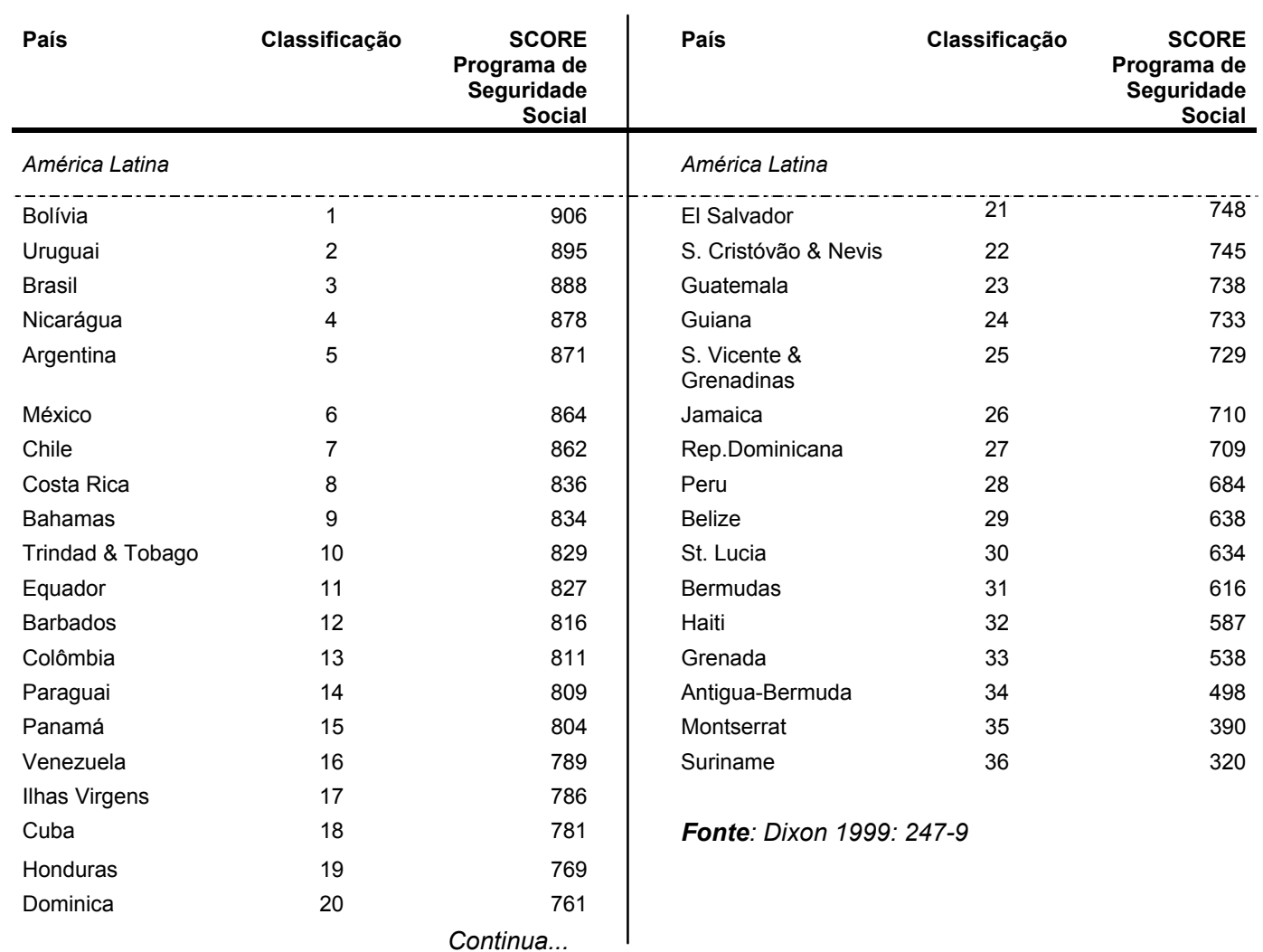




\section{Classificações Sub-regionais}

As classificações sub-regionais latino-americanas revelam variações muito amplas (Anexo 2). As regiões com o sistema de seguridade de mais alto padrão é a América Central, que é muito marginalmente melhor que aqueles obtidos na América do Sul, embora possam ser encontrados ali os sistemas de seguridade melhor planejados. No outro extremo, o Caribe tem os padrões mais pobres.

\section{América Central}

Nicarágua situa-se no topo da classificação da região, com um padrão de seguridade similar aos países da Europa Oriental-Hungria e Georgia; é pouco melhor que o do Canadá, e um pouco pior que o do Reino Unido. A força de seu modelo é o programa para incapacitados (classificado em $6^{\circ}$ lugar, junto com Brasil e Suécia). Claramente atrás está o México ( $2^{\circ}$ lugar) e Costa Rica $\left(3^{\circ}\right)$ ambos destacando-se com relação ao resto da sub-região. Ao final da classificação está Belize ( $8^{\circ}$ e último lugar), Guatemala $\left(7^{\circ}\right)$ e El Salvador $\left(6^{\circ}\right)$, todos eles, entretanto, obtendo um padrão consideravelmente melhor que os sistemas mais pobres do Caribe e América do Sul.

\section{América do Sul}

Bolívia tem o sistema melhor concebido desta sub-região, seguida um pouco atrás pelo Uruguai ( $2^{\circ}$ lugar) e Brasil $\left(3^{\circ}\right)$. A força do modelo boliviano está nos arranjos de financiamento, no contexto de um padrão global que o coloca entre o primeiro terço de países na sub-região. Uruguai apresenta as melhores pensões e benefícios de programas voltados para maternidade, desemprego e saúde. No Brasil, os programas de acidentes de trabalho e de invalidez se destacam (ambos equivalentes ao $6^{\circ}$ e $7^{\circ}$ programas melhor concebidos em suas categorias no mundo, respectivamente).Chile tem programas de auxílio-família de classificação mundial ( $2^{\mathrm{a}}$ colocação mundial). $\mathrm{Na}$ base da classificação sul-americana, acima do Suriname, está o Peru $\left(11^{\circ}\right)$ que, de forma intrigante, tem um programa de auxílio-doença destacado (equivalente ao $8^{\circ}$ do mundo), Guiana $\left(10^{\circ}\right)$ e Venezuela $\left(9^{\circ}\right)$.

\section{$\underline{\text { Caribe }}$}

Esta é a região mais débil na América Latina por uma margem considerável. Apenas se destacam as Bahamas, que tem o melhor sistema de seguridade na região, e Trinidad \& Tobago ( $2^{\circ}$ lugar), e, em um grau menor, Barbados $\left(3^{\circ}\right)$. No outro extremo, seis países ficam abaixo dos padrões mínimos estabelecidos para a América do Sul (com base no Peru) e para a América Central (com base em Belize). De fato, Montserrat $\left(16^{\circ} \mathrm{e}\right.$ último) compete com Suriname a presença de um dos mais pobres sistemas de seguridade do mundo. 


\section{Classificações de Programas}

\section{Programas para Idosos}

Nicarágua tem o melhor programa para idosos na América Latina, seguido muito de perto pelo Brasil $\left(2^{\circ}\right)$, e com Costa Rica $\left(3^{\circ}\right)$ um pouco atrás. O Haiti apresenta por uma margem considerável o programa mais pobre, atrás do Suriname.

\section{Programas de Invalidez}

Brasil e Nicarágua compartilham e claramente lideram o campo latino americano com programas para invalidez equivalentes ao $6^{\circ}$ lugar no mundo (com a Suécia), e que é consideravelmente melhor que seu rival próximo, Argentina $\left(3^{\circ}\right)$. O Haiti novamente apresenta o pior programa, logo abaixo de Montserrat e Santa Lucia.

\section{Programas de Pensões}

Sem qualquer dúvida, o Uruguai tem o melhor programa de benefícios na América Latina, seguido por um conjunto composto da Costa Rica $\left(2^{\circ}\right)$, Brasil $\left(3^{\circ}\right)$ e Bolívia $\left(4^{\circ}\right)$. Antigua tem o pior programa, logo abaixo de Bermudas.

\section{Programa de Auxílio-doença}

Peru, com seu programa de classificação mundial (classificação equivalente ao $8^{\circ}$ lugar no mundo, junto com Noruega) está logo à frente do Brasil e México (equivalente ao $2^{\circ}$ ) no âmbito latino-americano. A Guiana tem o programa mais pobre, ainda pior que o conjunto formado por Panamá, Dominicanas, Equador, S.Cristóvão \& Névis, Santa Lucia e São Vicente \& Grenadinas.

\section{Auxílio-Maternidade}

Uruguai está bem à frente de seus riveis mais próximos, Peru (2\%lugar) e Cuba $\left(3^{\circ}\right)$. Colômbia tem o pior programa.

\section{Programas de Auxílio- Invalidez temporária por trabalho}

Três países dominam essa categoria, cada um tendo um programa classificado entre os 10 primeiros em termos globais: Brasil e Cuba (equivalente ao $6^{\circ}$ lugar, com Lituânia e Eslováquia), e Trinidad \& Tobago (equivalente ao $10^{\circ}$ lugar, com Estônia, França, Hungria, Luxemburgo. Rússia e lugoslávia). Honduras tem o pior programa, logo abaixo da República Dominicana.

\section{Programa de Auxílio- Invalidez permanente por trabalho}

Brasil também lidera essa categoria, tendo um programa equivalente ao $9^{\circ}$ lugar no mundo (com Malásia, Rússia e Espanha).Em seguida estão Costa Rica e El Salvador (equivalente ao $2^{\circ}$ lugar). A República Dominicana tem o pior programa, algo abaixo de São Vicente \& Grenadinas. 


\section{Programa de Aposentadoria por Serviço}

O México se destaca com um programa equivalente ao $6^{\circ}$ no mundo ( com Alemanha e Moldova), calaramente à frente de Costa Rica ( $2^{\circ}$ na América Latina) e Equador $\left(3^{\circ}\right)$. A República Dominicana tem o pior programa, bem marginalmente abaixo de Honduras.

\section{Programa de Auxílio-Desemprego}

Dos 12 países com esses programas, Uruguai apresenta o melhor, logo à frente da Argentina $\left(2^{\circ}\right)$. No outro extremo está Equador, logo à frente da Guatemala.

\section{Programas de Auxílio-Família}

Dos 10 países que possuem esse programa, Chile é de longe o melhor concebido, classificado em $2^{\circ}$ lugar no mundo, junto com a França. Costa Rica está no outro extremo.

\section{Programa de Auxílio-Saúde}

Uruguai tem o melhor programa na América Latina, à frente de seus rivais mais próximos, Argentina e Venezuela (equivalentes ao $2^{\circ}$ lugar). Montserrat tem claramente o pior programa, logo abaixo do Haiti.

\section{Conclusão}

Este artigo procurou ampliar a literatura sobre seguridade social através da incorporação da dimensão avaliativa. Até agora, apenas Kaim-Caudle (1973) enfrentou os desafios epistemológicos e metodológicos da avaliação comparativa dos sistemas de seguridade social. Essa relutância em engajar-se na pesquisa comparativa-avaliativa não é tão surpreendente, dado que a inclinação neopositivista entre os cientistas sociais deprecia, se não condena, qualquer metodologia avaliativa que não se restrinja às comparações com aqueles países com os quais há dados sobre sistema de seguridade social ou dados de desempenho estritamente comparáveis. Essa perspectiva é difícil sustentar, porque, no limite, a disponibilidade de dados compatíveis e confiáveis constrange o avaliador. A avaliação de programa - avaliação do desenho legal estabelecido - é o único enfoque possível em uma escala global ou regional. A metodologia de avaliação adotada envolveu julgamentos qualitativos com base no conjunto de premissas valorativas extraídas das convenções da OIT, segundo padrões mínimos de seguridade social.

A conclusão apresentada é que ao nível sub-regional, a América Central tem os mais altos padrões, marginalmente à frente da América do Sul. Os melhores programas de seguridade social na América Latina, entretanto, podem ser observados sobretudo na América do Sul - Bolívia $\left(1^{\circ}\right)$, Uruguai $\left(2^{\circ}\right)$, Brasil $\left(3^{\circ}\right)$ e Argentina $\left(4^{\circ}\right)$. Programas de Seguridade Social com aspectos de padrão mundial existem no Brasil (programa de auxílio-invalidez e acidentes de trabalho), Argentina (programa de auxílio-família), Chile 
(programa de auxílio-família), Peru (programa de auxílio-doença) e Nicarágua (programa de auxílio-invalidez).

\section{BIBLIOGRAFIA}

DIXON, J. (1999). Social Security In Global Perspective. Westport, CT: Praeger.

DIXON, J. (1998). "Comparative Social Security: The Challenge of Evaluation." Journal of Comparative Policy Analysis, 1(1): 66-94.

DIXON, J. and SCHEURELL, R. P.(eds.) (1990), Social Welfare in Latin America, London: Croom Helm.

International Labor Organisation (ILO) (1952a). Social Security (Minimum Standards) Convention, 1952 (No. 102). Geneva: ILO.

ILO (1952b). Maternity Protection Convention (Revised. 1952, (No. 103). Geneva: ILO.

ILO (1964). Employment Injury Benefits Convention, 1964 (No. 121). Geneva: ILO.

ILO (1966). Revision of Conventions Nos. 35, 36, 37, 3839 and 40 Concerning Old-Age, Invalidity and Survivors' Pensions (Report V, Part 1, International Labor Conference, 50th Session). Geneva: ILO.

ILO (1967). Invalidity, Old-Age and Survivors' Benefits Convention, 1967 (No. 128). Geneva: ILO.

ILO (1968). Revision of Conventions Nos. 24 and 25 Concerning Sickness Insurance (Report VI, Part 1, International Labor Conference, 52nd Session). Geneva: ILO.

ILO (1969). Medical Care and Sickness Benefits Convention, 1969 (No. 130). Geneva: ILO.

ILO (1972a). "Social Security in Latin America: Evolution and Prospects." International Social Security Review 25 (3): 305-56.

ILO (1972b). The Cost of Social Security. Geneva: ILO.

ILO (1988). Employment Promotion and Protection Against Unemployment Convention, 1988 (No. 168). Geneva: ILO.

ILO 1989. Social Security Protection in Old-Age. Geneva: ILO.

ILO 1993. Report of the Tripartite Regional Meeting of Experts on Social Security in the Americas (GB 258/ESP/7/7). Geneva: ILO.

International Social Security Association (ISSA) (1976). Methods of Evaluating the Effectiveness of Social Security Programmes (Studies and Research 8). Geneva: ISSA. 
KAIM-CAUDLE, P. R. (1973). Comparative Social Policy and Social Security. London: Martin Robertson.

McGREEVEY, W. (1990). Social Security in Latin America: Issues and Options for the World Bank (Discussion Paper 110). Washington, DC: World Bank.

MESA-LAGO, C. (1978). Social Security in Latin America: Pressure Groups, Stratification and Inequality. Pittsburgh: University of Pittsburgh Press.

MESA-LAGO, C. (1983). "Social Security and Extreme Poverty in Latin America." Journal of Economic Development 12 (1): 83-110.

MESA-LAGO, C. (ed.) 1985a. The Crisis of Social Security and Health Care. Pittsburgh: University of Pittsburgh Press.

MESA-LAGO, C. (1985b). "Alternative Strategies to the Social Security Crisis: Socialists, Market and Mixed Approaches." In MESA-LAGO, C. (ed.), The Crisis of Social Security and Health Care. Pittsburgh: University of Pittsburgh Press.

MESA-LAGO, C. (1986). "Comparative Study of the Development of Social Security in Latin America." International Social Security Review 39 (2): 127-51.

MESA-LAGO, C. (1988). "Social Insurance: The Experience of Three Countries in the Englishspeaking Caribbean." International Labor Review 127 (4): 479-96.

MESA-LAGO, C. (1991a). "Formal Social Security in Latin America and the Caribbean." In AHMED, E. and DREZE, J. (eds.), Social Security in Developing Countries. Oxford: Clarendon Press.

MESA-LAGO, C. (1991b). Social Security and Prospects for Equity in Latin America (Discussion Paper 140). Washington, DC: World Bank.

MESA-LAGO, C. (1992a). Social Security in Latin America: Issues and Options for the World Bank. Pittsburgh: University of Pittsburgh Press.

Mesa-Lago, C. (1992b). Ascent to Bankruptcy, Financing Social Security in Latin America. Pittsburgh: University of Pittsburgh Press.

Mesa-Lago, C. (1994). Changing Social Security in Latin America: Towards Alleviating the Social Cost of Economic Reform. Boulder, CO: Lynne Rienner.

OECD (Organization for Economic Co-operation and Development) (1976). Public Expenditure on Income Maintenance Programmes (Studies in Resource Allocation). Paris: OECD.

OECD (1977). The Tax/Benefit Position of Selected Income Groups in OECD Member Countries in 1971-76. Paris: OECD.

OTTING, A. (1993). "International Labor Standards: A Framework for Social Security." in International Labor Review, 132 (1): 169-83.

PAILLAS, C. A. (1979). "Pensions in Latin America: The Present Situation." International Social Security Review 32 (3): 288-303.

ROSE, R. (1995). "Making Progress and Catching up: Comparative Analysis for Social Policymaking." International Social Science Journal, 47 (1): 113-25. 
RYS, V. 1966. "Comparative Studies of Social Security: Problems and Perspectives." Bulletin of the International Social Security Bulletin, 19 (3): 242-68.

TAMBURI, G. (1981). The International Labor Organization and the Development of Social Insurance (Social Security Department Working Paper). Geneva: ILO.

TAMBURI, G. (1985). "Social Security in Latin America: Trends and Outlook." In: MESA-LAGO, C. (ed.), The Crisis of Social Security and Health Care. Pittsburgh: University of Pittsburgh Press. United States of America, Social Security Administration (US SSA) 1996. Social Security Programs Throughout the World - 1995. Washington, DC: US Government Printing Office.

World Bank 1995. "Social Security Administration in Latin America.", mimeo. Washington, DC. 
OPINIÃO PÚBLICA, Campinas, Vol.VI, $n^{\circ} 2,2000, p p .263-281$

ANEXO 1: Estratégias Principais de Seguridade Social por Programa, América Latina - 1995

\begin{tabular}{|c|c|c|c|c|c|c|c|c|c|c|c|c|}
\hline \multirow[t]{2}{*}{ Região/País } & \multicolumn{12}{|c|}{ Programas: } \\
\hline & Idosos & Invalidez & Morte & Doença & Maternidade & $\begin{array}{l}\text { Invalidez } \\
\text { Temporária } \\
\text { trabalho }\end{array}$ & $\begin{array}{l}\text { Invalidez } \\
\text { Permanente } \\
\text { Trabalho }\end{array}$ & $\begin{array}{c}\text { Aposentadoria } \\
\text { trabalho }\end{array}$ & $\begin{array}{l}\text { Desem- } \\
\text { prego }\end{array}$ & Familia & Crianças & Saúde \\
\hline \multicolumn{13}{|l|}{$\begin{array}{l}\text { AMERICA } \\
\text { CENTRAL }\end{array}$} \\
\hline BELIZE & A & A & A & A & A & A & A & & & & & 1 \\
\hline COSTA RICA & A & A & A & A & A & D & D & D & & A & & A \\
\hline EL SALVADOR & A & A & A & A & A & A & A & A & & & & A \\
\hline GUATEMALA & A & A & A & A & A & A & A & & $\mathrm{F}$ & & & A \\
\hline HONDURAS & A & A & A & A & A & A & A & A & D & & & A \\
\hline MÉXICO & A & A & A & A & A & A & A & A & D & & & A \\
\hline NICARÁGUA & A & A & A & A & A & A & A & A & & & A & 1 \\
\hline PANAMÁ & A & A & A & A & A & D & D & D & & & & A \\
\hline \multicolumn{13}{|l|}{ CARIBE } \\
\hline $\begin{array}{l}\text { ANTIGUA- } \\
\text { BARBUDA }\end{array}$ & A & A & A & A & A & & & & & & & 1 \\
\hline BAHAMAS & A & A & A & A & A & A & A & A & & & & I \\
\hline BARBADOS & A & A & A & A & A & A & A & A & A & & & 1 \\
\hline BERMUDAS & A & A & A & & & D & D & D & & & & $\mathrm{J}$ \\
\hline ILHAS VIRGENS & A & A & A & A & A & A & A & A & & & & A \\
\hline CUBA & A & A & A & A & A & A & A & A & & & & 1 \\
\hline DOMINICA & A & A & A & A & A & D & D & D & & & & I \\
\hline $\begin{array}{l}\text { REP. } \\
\text { DOMINICANA }\end{array}$ & A & A & A & A & A & A & A & A & & & & A \\
\hline GRENADA & A & A & A & A & A & & & & & & & 1 \\
\hline HAITI & A & A & A & & & A & A & A & & & & A \\
\hline JAMAICA & A & A & A & & D & A & A & A & & & & 1 \\
\hline MONTSERRAT & E & E & E & & & & & & & & & I \\
\hline $\begin{array}{l}\text { S.CRISTÓVÃO } \\
\text { \& NÉVIS }\end{array}$ & A & A & A & A & A & A & A & A & & & & 1 \\
\hline
\end{tabular}


Sistemas de Seguridade Social na América Latina

\begin{tabular}{|c|c|c|c|c|c|c|c|c|c|c|c|c|}
\hline \multirow[t]{2}{*}{ Região/País } & \multicolumn{12}{|c|}{ Programas: } \\
\hline & Idosos & Invalidez & Morte & Doença & Maternidade & $\begin{array}{l}\text { Invalidez } \\
\text { Temporária } \\
\text { trabalho }\end{array}$ & $\begin{array}{l}\text { Invalidez } \\
\text { Permanente } \\
\text { Trabalho }\end{array}$ & $\begin{array}{c}\text { Aposentadoria } \\
\text { trabalho }\end{array}$ & $\begin{array}{l}\text { Desem- } \\
\text { prego }\end{array}$ & Familia & Crianças & Saúde \\
\hline STA LUCIA & A & A & A & A & A & A & A & & & & & 1 \\
\hline $\begin{array}{l}\text { S. VICENTE } \\
\& \\
\text { GRENADINAS }\end{array}$ & A & A & A & A & A & D & D & D & & & & 1 \\
\hline $\begin{array}{l}\text { TRINIDAD \& } \\
\text { TOBAGO }\end{array}$ & A & A & A & A & A & A & A & A & & & & 1 \\
\hline \multicolumn{13}{|l|}{$\begin{array}{l}\text { AMÉRICA DO } \\
\text { SUL }\end{array}$} \\
\hline ARGENTINA & A & A & A & D & A & D & D & D & A & A & A & A \\
\hline BOLIVIA & A & A & A & A & A & A & A & A & D & & D & A \\
\hline$B R A S I L$ & A & A & A & A & A & A & A & A & B & & A & A \\
\hline CHILE & G & G & G & A & A & A & A & A & c & c & c & 1 \\
\hline COLÔMBIA & A & A & A & A & A & A & A & A & $\mathrm{F}$ & & A & A \\
\hline$E Q \cup A D O R$ & A & A & A & A & A & A & A & A & A & & & A \\
\hline GUIANA & A & A & A & A & A & A & A & A & & & & 1 \\
\hline PARAGUAI & A & A & A & A & A & A & A & A & & & D & A \\
\hline PERU & G & G & G & A & A & A & A & A & & & & A \\
\hline SURINAME & A & & & & & & & & & & c & $\mathrm{i}$ \\
\hline URUGUAI & A & A & A & A & A & D & D & D & A & & A & A \\
\hline VENEZUELA & A & A & A & A & A & A & A & A & A & & & A \\
\hline
\end{tabular}

Fonte: Dixon 1999: 25-26
Legenda:

A Seguro social;

F Programa Legal Recursos Pessoais

Contribuições Sociais

D Impostos/Taxas do Empregador

G Programa Legal de Pensões Pessoai

E Fundo Nacional Previdenciário

I Sistema Público de Saúde

J Sistema Nacional de Seguro Saúde 
OPINIÃO PÚBLICA, Campinas, Vol.VI, $n^{\circ} 2,2000, p p .263-281$

ANEXO 2: Scores - Sistema de Seguridade Social, 1995

REGIÃO/PAÍS

AMÉRICA CENTRAL

\begin{tabular}{|c|c|c|c|c|c|c|c|c|c|c|c|c|c|c|c|}
\hline BELIZE & 82 & 70 & 75 & 80 & 82 & 95 & 102 & & & & 82 & 100 & 69 & 61 & 638 \\
\hline COSTA RICA & 112 & 93 & 100 & 88 & 83 & 108 & 118 & 123 & & 14 & 87 & 100 & 74 & 84 & 836 \\
\hline EL SALVADOR & 88 & 88 & 63 & 83 & 83 & 102 & 118 & 93 & & & 60 & 100 & 87 & 71 & 748 \\
\hline GUATEMALA & 97 & 107 & 82 & 80 & 87 & 100 & 87 & & 63 & & 69 & 100 & 85 & 70 & 738 \\
\hline HONDURAS & 83 & 77 & 57 & 82 & 78 & 80 & 98 & 83 & 68 & & 84 & 100 & 96 & 72 & 769 \\
\hline MEXICO & 92 & 88 & 90 & 102 & 87 & 112 & 115 & 128 & 68 & & 75 & 105 & 77 & 87 & 864 \\
\hline NICARAGUA & 119 & 120 & 85 & 82 & 85 & 105 & 105 & 112 & & 58 & 73 & 100 & 95 & 86 & 878 \\
\hline PANAMA & 98 & 97 & 63 & 72 & 80 & 108 & 107 & 118 & & & 83 & 100 & 102 & 75 & 804 \\
\hline
\end{tabular}

CARIBE

ANTIGUA-BARBUDA

$80-63$

55

BAHAMAS

BARBADOS

BERMUDA

ILHAS VIRGENS

CUBA

DOMINICA

REP. DOMINICANA

$\begin{array}{rrrrrrrrr}102 & 92 & 92 & 95 & 87 & 100 & 112 & 103 & \\ 87 & 77 & 73 & 78 & 87 & 101 & 110 & 102 & 67 \\ 82 & 60 & 58 & & & 98 & 103 & 97 & \\ 78 & 67 & 82 & 80 & 85 & 105 & 113 & 105 & \\ 92 & 85 & 83 & 97 & 92 & 118 & 113 & 110 & \\ 92 & 77 & 72 & 73 & 77 & 98 & 107 & 102 & \\ 99 & 102 & 78 & 78 & 78 & 83 & 75 & 82 & \end{array}$

$\begin{array}{rrrrr}75 & 100 & 92 & 39 & 498 \\ 75 & 100 & 108 & 78 & 834 \\ 82 & 100 & 92 & 78 & 816 \\ 82 & 100 & 96 & 53 & 616 \\ 75 & 100 & 108 & 72 & 786 \\ 83 & 95 & 66 & 79 & 781 \\ 73 & 100 & 101 & 70 & 761 \\ 65 & 105 & 79 & 67 & 709\end{array}$


Sistemas de Seguridade Social na América Latina

\begin{tabular}{|c|c|c|c|c|c|c|c|c|c|c|c|c|c|c|c|c|}
\hline & & 1 & 2 & 3 & 4 & 5 & 6 & 7 & 8 & 9 & 10 & 11 & 12 & 13 & 14 & 15 \\
\hline GRENADA & & 82 & 70 & 72 & 83 & 80 & & & & & & 68 & 100 & 105 & 41 & 538 \\
\hline HAITI & & 58 & 52 & 60 & & & 99 & 100 & 90 & & & 61 & 100 & 106 & 47 & 587 \\
\hline JAMAICA & & 73 & 70 & 70 & & 82 & 105 & 113 & 94 & & & 70 & 105 & 110 & 62 & 710 \\
\hline MONTSERRAT & & 70 & 57 & 75 & & & & & & & & 58 & 100 & 101 & 24 & 390 \\
\hline S.CRISTÓVÃO \& NÉVIS & & 87 & 77 & 72 & 73 & 78 & 97 & 110 & 97 & & & 78 & 100 & 91 & 70 & 745 \\
\hline STA LUCIA & & 75 & 57 & 65 & 73 & 77 & 97 & 98 & & & & 77 & 100 & 90 & 56 & 634 \\
\hline S. VICENTE \& GRENADINAS & & 82 & 70 & 62 & 73 & 77 & 100 & 82 & 98 & & & 78 & 100 & 103 & 66 & 729 \\
\hline TRINIDAD \& TOBAGO & & 97 & 88 & 80 & 92 & 93 & 117 & 108 & 118 & & & 73 & 100 & 99 & 79 & 829 \\
\hline & 1 & & & 3 & 4 & 5 & 6 & 7 & 8 & 9 & 10 & 11 & 12 & 13 & 14 & 15 \\
\hline
\end{tabular}

AMÉRICA DO SUL

\begin{tabular}{|c|c|c|c|c|c|c|c|c|c|c|c|c|c|c|c|}
\hline ARGENTINA & 85 & 113 & 88 & 90 & 85 & 105 & 102 & 107 & 85 & 95 & 95 & 105 & 37 & 95 & 871 \\
\hline BOLIVIA & 108 & 85 & 95 & 88 & 90 & 112 & 112 & 110 & 73 & 63 & 80 & 97 & 79 & 92 & 906 \\
\hline BRASIL & 117 & 120 & 97 & 102 & 87 & 118 & 125 & 118 & 77 & 61 & 81 & 97 & 25 & 100 & 888 \\
\hline CHILE & 77 & 100 & 82 & 97 & 85 & 110 & 112 & 100 & 73 & 108 & 93 & 92 & 42 & 94 & 862 \\
\hline COLOMBIA & 108 & 110 & 98 & 65 & 73 & 102 & 98 & 107 & 67 & 34 & 76 & 105 & 51 & 85 & 811 \\
\hline EQUADOR & 100 & 73 & 92 & 73 & 87 & 97 & 112 & 122 & 62 & & 74 & 100 & 87 & 81 & 827 \\
\hline GUIANA & 75 & 80 & 73 & 70 & 78 & 87 & 103 & 112 & & & 82 & 100 & 87 & 69 & 733 \\
\hline PARAGUAI & 100 & 82 & 88 & 80 & 78 & 98 & 102 & 102 & & 56 & 70 & 100 & 91 & 78 & 809 \\
\hline PERU & 70 & 75 & 82 & 105 & 92 & 103 & 103 & 95 & & & 62 & 100 & 41 & 72 & 684 \\
\hline SURINAME & 65 & & & & & & & & & 56 & 43 & 100 & 101 & 15 & 320 \\
\hline URUGUAI & 107 & 95 & 110 & 90 & 102 & 100 & 102 & 110 & 88 & 60 & 97 & 100 & 49 & 96 & 895 \\
\hline VENEZUELA & 100 & 93 & 88 & 77 & 75 & 93 & 95 & 100 & 70 & & 95 & 90 & 66 & 81 & 789 \\
\hline
\end{tabular}

Fonte: Dixon 1999: 260-1.

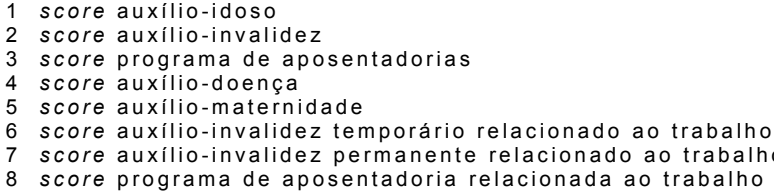

9 score programa desemprego

10 score auxílio-família

11 score programa de benefício

12 score para administração

4 score médio do programa

15 score do programa de Seguridade Socia 